\title{
USO DE SUPLEMENTOS ALIMENTARES NO MANEJO NUTRICIONAL EM IDOSOS COM SARCOPENIA
}

\section{USE OF FOOD SUPPLEMENTS IN NUTRITIONAL MANAGEMENT IN OLDER PEOPLE WITH SARCOPENIA}

\author{
Gisele Viana de Moura ${ }^{1}$, Mateus Cunha de Sousa ${ }^{1}$, Palloma de Sousa ${ }^{1}$, Carlos Henrique \\ Ribeiro Lima². \\ Faculdade Estácio de Teresin ${ }^{1}$, Universidade Federal do Piauí ${ }^{2}$
}

\begin{abstract}
OBJECTIVE: To analyze the effect of dietary supplements used in the treatment and prevention of sarcopenia in the elderly. METHODOLOGY: Integrative literature review on the relationship between the use of dietary supplements and sarcopenia in the elderly. Electronic databases used: National Library of Medicine (Pubmed) and Medical Literature Analysis and Retrieval System Online (Medline) in March 2021. Then 8 articles were selected for the development of the results of this research, dealing with randomized studies, case studies, placebo-controlled research and prospective cohort studies. RESULTS AND DISCUSSION: Most studies have demonstrated scientific evidence on the use of dietary supplements in improving body composition in the elderly, in improving the relative skeletal mass index, handgrip strength, in physical composition and significantly improving some criteria of sarcopenia in the elderly, as functional performance measured by walking time and improvement in lean mass index in active elderly. FINAL CONSIDERATIONS: Protein supplementation, in elderly people with sarcopenia, improves muscle strength and thickness combined with physical exercise. In addition, the consumption of whey protein, creatine and leucine bring health benefits by improving the quality of life. In addition, physical activity is also effective in preventing and restoring muscle loss.
\end{abstract}

Keywords: Sarcopenia, Nutritional Supplementation, Quality of Life, Elderly.

\section{Resumo}

OBJETIVO: Analisar o efeito de suplementos alimentares utilizados no tratamento $e$ prevenção de sarcopenia em idosos. METODOLOGIA: Revisão integrativa da literatura sobre a relação do uso de suplementos alimentares e a sarcopenia em idosos. Bases eletrônicas utilizadas: National Library of Medicine (Pubmed) e Medical Literature Analysis and Retrieval System Online (Medline) em março de 2021. Foram selecionados então 8 artigos para o desenvolvimento dos resultados dessa pesquisa, tratando-se de estudos randomizados, estudos de casos, pesquisas controladas por placebo e estudos de coorte prospectivo. RESULTADOS E DISCUSSÃO: A maioria dos estudos demonstraram evidências cientificas no uso de suplementos alimentares na melhora da composição corporal nos idosos, na melhora o índice de massa esquelética relativa, força de preensão manual, na composição física $e$ melhora significativamente alguns critérios da sarcopenia em idosos, como desempenho funcional medido pelo tempo de caminhada $e$ melhora do índice de massa magra em idosos ativos. CONSIDERAÇÕES FINAIS: A suplementação de proteína, em idosos com sarcopenia, melhora a força e espessura muscular combinado com o exercício físico, além disso, o consumo de whey protein, creatina e leucina trazem benefícios para saúde melhorando a qualidade de vida. Ademais, a atividade física também se mostra eficaz na prevenção e restauração de perda muscular.

Palavras-chave: Sarcopenia, Suplementação Nutricional, Qualidade de Vida, Idoso. 
Sarcopenia, termo grego que significa "pobreza da carne", é a perda progressiva e generalizada de massa muscular, função, qualidade e força impulsionada pelo processo de envelhecimento. Essa perda de massa muscular geralmente leva à diminuição da força e dos níveis de atividade e pode contribuir para problemas de mobilidade, osteoporose, quedas e fraturas, fragilidade e perda da função física e independência. ${ }^{1}$

Inúmeros fatores relacionados à idade estão envolvidos no desenvolvimento da sarcopenia, dentre esses fatores encontra-se a degeneração neuromuscular, alterações na renovação da proteína muscular, alterações nos níveis e sensibilidade hormonais, inflamação crônica, estresse oxidativo e fatores de estilo de vida. $^{2}$

Estima-se que a sarcopenia afete $30 \%$ das pessoas com mais de 60 anos e mais de $50 \%$ das pessoas com mais de 80 anos. Entre as idades de 30 e 60, o adulto médio ganhará $1 \mathrm{~kg}$ de peso e perderá $1 / 2 \mathrm{~kg}$ de músculo anualmente. Após os 70 anos, a perda muscular acelera para $15 \%$ por década. Pessoas obesas e também com sarcopenia (obesidade sarcopênica) parecem ter resultados piores do que aqueles que não são obesos. ${ }^{3}$

Há um declínio significativo na ingestão alimentar e energética com o aumento da idade, à medida que as necessidades energéticas diminuem, chegando a uma queda média de cerca de $25 \%$ entre as idades de 40 e 70 anos. A baixa ingestão de alimentos e dietas monótonas colocam os idosos em risco porque, à medida que a ingestão total de alimentos diminui, para a maioria dos nutrientes ocorre um declínio correspondente na ingestão. Sendo um desafio para os Nutricionistas adequar todos os nutrientes necessários para o pleno funcionamento do organismo idoso através apenas da alimentação, muitas vezes sendo essencial recorrer ao uso da suplementação nutricional. ${ }^{4}$

Os principais estudos que abordam o tema, trata a suplementação nutricional com proteína de soro de leite, aminoácidos essenciais e vitamina $D$, em conjunto com atividade física, como a principal forma de tratamento da sarcopenia, pois contribuem para o aumento da massa magra e força muscular de forma que, a nutrição seja um elemento-chave das intervenções multimodais para fragilidade e sarcopenia. ${ }^{5}$
Recomenda-se uma ingestão adequada de proteínas no tratamento da sarcopenia induzida pela perda de peso em pessoas com obesidade sarcopênica. ${ }^{6}$ A creatina quando ingerida durante $o$ treinamento de resistência, geralmente, tem o potencial de aumentar a massa e o desempenho muscular, dessa forma, influencia o metabolismo do fosfato de alta energia e a absorção de cálcio. ${ }^{7}$

0 presente estudo tem como objetivo analisar na literatura o efeito de suplementos alimentares utilizados no tratamento e prevenção de sarcopenia em idosos.

\section{Metodologia}

O presente estudo trata-se de uma revisão integrativa da literatura sobre a relação do uso de suplementos alimentares e a sacorpenia em idosos. O material foi acessado por meio das bases eletrônicas National Library of Medicine (Pubmed) e Medical Literature Analysis and Retrieval System Online (Medline) em março de 2021. Foram usados os seguintes descritores e operadores booleanos português e inglês: "Sarcopenia e Suplementação nutricional" (Sarcopenia and Nutritional Supplementation) $e$ "Sarcopenia e nutrição" (Sarcopenia AND nutrition).

Os critérios de seleção dos artigos foram feitos pelo ano de publicação do trabalho, incluindo as pesquisas publicadas entre 2016 a 2020, pelos idiomas português e inglês, e pelo conteúdo do estudo na qual relacionasse os suplementos alimentares e sua relação com a sarcopenia em idosos.

Após ser realizada a busca dos estudos foi encontrado um total de 817 artigos. Os artigos selecionados foram analisados para verificar se atendiam aos critérios de inclusão, aqueles que expuseram um estudo delineado, completo e com resultados eficazes foram inclusos, foram descartados aqueles estudos que se tratavam de resenhas, comentários, textos que estudava a história e a termologia da palavra sarcopenia, trabalhos realizados a partir de entrevistas, artigos repetidos ou que não contemplassem os critérios predefinidos. Foram selecionados então 8 artigos para 0 desenvolvimento dos resultados dessa pesquisa, tratando-se de estudos randomizados, estudos de casos, pesquisas controladas por placebo e estudos de coorte prospectivo. Na figura1 encontra-se o total de artigos encontrados, excluídos e selecionados. 
Figura1 - Fluxograma do total de artigos encontrados, excluídos, elegíveis e selecionados.

Total de artigos
encontrado
817

Total de artigos
excluidos com
bases no critério
de exclusão
809

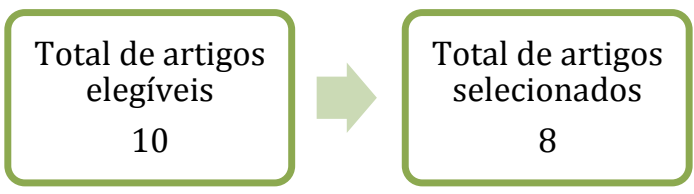

A busca dos artigos foi feita por meio da estratégia PICO em que cada letra corresponde ao seguinte componente: P: Participantes, I: Intervenção, C: Comparação, O: Desfecho.

Tabela 1 - Termos de busca utilizada no processo de investigação e seleção dos artigos. Teresina, PI, Brasil. 2021.

\begin{tabular}{|c|c|c|c|c|}
\hline DESCRIÇÃO & PICO & TEMA & $\begin{array}{c}\text { TERMOS DE } \\
\text { BUSCA }\end{array}$ & TIPO \\
\hline Participantes & $\mathrm{P}$ & Idoso, Sarcopenia. & "and" & $\begin{array}{l}\text { Artigos de } \\
\text { periódicos. }\end{array}$ \\
\hline Intervenção & I & $\begin{array}{c}\text { Suplementação } \\
\text { Nutricional. }\end{array}$ & "and" & $\begin{array}{l}\text { Artigos de } \\
\text { periódicos. }\end{array}$ \\
\hline Comparação & $\mathrm{C}$ & Qualidade de Vida. & "and" & $\begin{array}{l}\text { Artigos de } \\
\text { periódicos. }\end{array}$ \\
\hline Desfecho & 0 & $\begin{array}{c}\text { Idoso, } \\
\text { Suplementação } \\
\text { Nutricional. }\end{array}$ & "and" & $\begin{array}{l}\text { Artigos de } \\
\text { periódicos. }\end{array}$ \\
\hline
\end{tabular}

Fonte: Dados da pesquisa, 2021.

\section{Resultados}

Na tabela 2 pode-se observar a distribuição dos artigos, conforme o autor, ano, objetivo, tipo de estudo e resultados. 
Tabela 2 - Distribuição das produções científicas sobre o uso de suplementos alimentares na prevenção da sarcopenia em idosos, publicadas no período de 2016 a 2020 segundo o autor, ano, objetivo, metodologia e principais resultados.

\begin{tabular}{|c|c|c|c|}
\hline Autor/ano & Objetivo & Metodologia & Resultados \\
\hline $\begin{array}{c}\text { Martínez et al, } \\
2020 .\end{array}$ & $\begin{array}{l}\text { Avaliar a eficácia da } \\
\text { administração de } \\
\text { leucina em idosos com } \\
\text { sarcopenia }\end{array}$ & $\begin{array}{l}\text { Projeto duplo- } \\
\text { cego, } \\
\text { randomizado e } \\
\text { controlado por } \\
\text { placebo }\end{array}$ & $\begin{array}{l}\text { Melhora significativamente alguns } \\
\text { critérios de sarcopenia em idosos, dessa } \\
\text { forma, o uso da suplementação de } \\
\text { leucina pode ter alguns efeitos benéficos } \\
\text { na sarcopenia e pode ser usado para o } \\
\text { tratamento da sarcopenia em indivíduos } \\
\text { mais velhos. }\end{array}$ \\
\hline $\begin{array}{l}\text { Björkman et } \\
\text { al, } 2020 \text {. }\end{array}$ & $\begin{array}{c}\text { Testar os efeitos a } \\
\text { longo prazo da } \\
\text { suplementação de } \\
\text { whey enriquecido com } \\
\text { soro do leite no } \\
\text { músculo e na } \\
\text { performance física em } \\
\text { idosos com sarcopenia }\end{array}$ & $\begin{array}{c}\text { Estudo } \\
\text { randomizado } \\
\text { duplo-cego. }\end{array}$ & $\begin{array}{l}\text { Os suplementos de teste não tiveram } \\
\text { efeitos significativos no desempenho } \\
\text { físico, as mudanças de } 12 \text { meses para } \\
\text { bateria de desempenho físico curto } \\
\text { foram - } 0,55,-, 05 \text { e } 0,03 \text { pontos nos } \\
\text { grupos controle, isocalórico e de } \\
\text { proteína. }\end{array}$ \\
\hline $\begin{array}{c}\text { Aas et al, } \\
2019 .\end{array}$ & $\begin{array}{c}\text { Conduzir um exame } \\
\text { completo da adaptação } \\
\text { ao treinamento de força } \\
\text { e suplementação de } \\
\text { proteína em idosos com } \\
\text { mobilidade limitada } \\
\text { indivíduos. }\end{array}$ & $\begin{array}{c}\text { Estudo } \\
\text { randomizado }\end{array}$ & $\begin{array}{c}\text { A massa magra da perna aumentou } \\
\text { desde a linha de base no ST }(0,7 \pm 0,3 \\
\mathrm{kg}) \text {, juntamente com o aumento da } \\
\text { espessura do vasto lateral } \\
(4,4 \pm 3,2 \%) \text {, reto femoral }(6,7 \pm 5,1 \%) \text { e } \\
\text { vasto intermediário }(5,8 \pm 5,9 \%) .\end{array}$ \\
\hline $\begin{array}{c}\text { Dolan et al, } \\
2019 .\end{array}$ & $\begin{array}{l}\text { Testar a suplementação } \\
\text { de creatina associada } \\
\text { ao exercício físico em } \\
\text { idosos sarcopênicos. }\end{array}$ & $\begin{array}{c}\text { Estudo } \\
\text { randomizado }\end{array}$ & $\begin{array}{l}\text { Pode ser uma estratégia alimentar eficaz } \\
\text { e deve ser usada para complementar os } \\
\text { benefícios do treinamento físico. }\end{array}$ \\
\hline $\begin{array}{c}\text { Bo Y et al, } \\
2018 .\end{array}$ & $\begin{array}{l}\text { Avaliar a utilização do } \\
\text { whey na preservação } \\
\text { da massa muscular em } \\
\text { idosos }\end{array}$ & $\begin{array}{l}\text { Pesquisa } \\
\text { controlada por } \\
\text { placebo }\end{array}$ & $\begin{array}{l}\text { Preservação da massa muscular, força e } \\
\text { qualidade de vida em idosos } \\
\text { sarcopênicos, em comparação com o } \\
\text { grupo placebo. }\end{array}$ \\
\hline $\begin{array}{l}\text { Park et al, } \\
2018 .\end{array}$ & $\begin{array}{l}\text { Investigar uma dose } \\
\text { efeito dependente da } \\
\text { suplementação de } \\
\text { proteína na massa } \\
\text { muscular e } \\
\text { fragilidade em idosos } \\
\text { pré-ferroviários ou }\end{array}$ & $\begin{array}{l}\text { Ensaio clínico } \\
\text { duplo-cego } \\
\text { controlado } \\
\text { randomizado }\end{array}$ & $\begin{array}{l}\text { A ingestão de proteínas de 1,5 g por kg } \\
\text { de peso tem os efeitos mais benéficos } \\
\text { em relação à prevenção da sarcopenia e } \\
\text { fragilidade em comparação com a } \\
\text { ingestão de proteínas de 0,8 e 1,2 g em } \\
\text { idosos frágeis ou pré-ferroviários em } \\
\text { risco de desnutrição. }\end{array}$ \\
\hline
\end{tabular}




\begin{tabular}{|c|c|c|c|}
\hline & frágeis desnutridos. & & \\
\hline $\begin{array}{l}\text { Malafarinaa } \\
\text { et al, } 2017 .\end{array}$ & $\begin{array}{c}\text { Avaliar } \\
\text { se a suplementação } \\
\text { nutricional oral } \\
\text { melhora a massa } \\
\text { muscular e os } \\
\text { marcadores } \\
\text { nutricionais } \\
\text { em pacientes idosos } \\
\text { com fratura de quadril. }\end{array}$ & $\begin{array}{c}\text { Estudo } \\
\text { randomizado }\end{array}$ & $\begin{array}{l}\text { Dos } 107 \text { pacientes randomizados (GI } \\
\text { n55, GC n52), } 49 \text { finalizaram o estudo no } \\
\text { GI e } 43 \text { no GC. IMC } \\
\text { e aLM foram estáveis nos pacientes do } \\
\text { GI, enquanto esses parâmetros } \\
\text { diminuíram no GC. }\end{array}$ \\
\hline $\begin{array}{c}\text { Rondanelli et } \\
\text { al, } 2016 .\end{array}$ & $\begin{array}{l}\text { Testar a hipótese de } \\
\text { que o suprimento } \\
\text { nutricional } \\
\text { com proteína de soro } \\
\text { de leite, aminoácidos } \\
\text { essenciais } \\
\text { e vitamina D melhora a } \\
\text { sarcopenia. }\end{array}$ & $\begin{array}{c}\text { Estudo } \\
\text { randomizado } \\
\text { duplo-cego, } \\
\text { controlado por } \\
\text { placebo. }\end{array}$ & $\begin{array}{c}\text { Comparado com atividade física e } \\
\text { placebo, a suplementação mais } \\
\text { atividade física aumentou a massa livre } \\
\text { de gordura, massa muscular esquelética } \\
\text { relativa, } \\
\text { distribuição de gordura e força de } \\
\text { preensão manual. }\end{array}$ \\
\hline
\end{tabular}

Fonte: Dados da pesquisa,

\section{Discussão}

A população estudada tratava-se de idosos com idades bem avançadas de 75 a 96 anos de idade, onde em sua maioria, eram mulheres $(68 \%){ }^{8}$ Os participantes não praticavam atividade física e não eram muito ativos, interferindo assim nos resultados, onde os suplementos de teste não tiveram efeitos significativos no desempenho físico e as mudanças em 12 meses no desempenho físico curto não foram tão significantes quando comparado os grupos de testes. Concluindo que, a suplementação de proteína enriquecida com soro de leite em combinação com exercícios físicos caseiros de baixa intensidade não atenuou a deterioração do desempenho muscular e físico em idosos com sarcopenia, desse modo, percebemos que a atividade física e o treinamento muscular provou ser o mais importante na prevenção e restauração de perda muscular nessas faixas etárias.

0 estudo foi realizado com 22 participantes dos sexos masculino e feminino com a faixa etária de idade entre 73 e 92 anos. Os participantes foram devidos em 2 grupos, um grupo que recebeu suplementação de proteína (ST), e o grupo que continuou com seus hábitos e atividades normais (CON). ${ }^{9}$ Os participantes que receberam suplementação de proteína melhoraram a espessura muscular, com isso, melhorando a espessura do vaso lateral, musculo reto femoral e respiratório, aumentaram a força muscular e desempenho funcional diminuindo o tempo para exultar o treinamento de elevação da cadeira e subida em escadas, já os participantes do grupo (CON) que não receberam suplementação, não apresentaram mudança na espessura muscular, mas tiveram um razoável aumento da massa muscular.

É possível observar que os participantes do estudo receberam suplementação de proteína a base de soro do leite, porém, não praticavam atividade física e isso mostrou que para aumentar a força muscular em idosos, a suplementação de proteína precisa estar associada com a atividade física. No entanto, os idosos do estudo que receberam suplementação de proteína e continuaram realizando suas 
atividades como elevação em cadeiras e subida em escadas, apresentaram um maior desempenho e força muscular.

Esse estudo randomizado teve a participação de 120 idosos com idade entre 70 e 85 anos com risco de desnutrição. ${ }^{10}$ Onde foi possível observar que a suplementação de proteína pode auxiliar no tratamento da sarcopenia. Porém, uma baixa ingestão como $(0,8 \mathrm{~g} / \mathrm{kg} / \mathrm{dia})$ não mostrou grandes resultados, mas os idosos que receberam a suplementação de $(1,2$ e $1,5 \mathrm{~g} / \mathrm{kg} / \mathrm{dia})$ apresentaram um aumento no peso, gordura, IMC, além de aumentar a velocidade de macha dos participantes.

Uma intervenção nutricional hiperproteica teve como participantes 198 idosos acima de 65 anos de idade internados com fratura no quadril e sarcopenia, dos 198 participantes 107 (52\%) foram randomizados. ${ }^{11}$ Esse estudo mostrou que, o uso de suplemento nutricional via oral auxiliou significativamente no tratamento de idosos com sarcopenia, e $80 \%$ dos idosos tiveram aderência a ingestão do suplemento. Com isso, os idosos que receberam suplementação de proteína via oral, apresentam um aumento na forma muscular e IMC, porém, os participantes que não receberam a suplementação apresentaram redução no IMC.

Diante disso, os estudos mostram que, a suplementação de proteína pode auxiliar no tratamento da sarcopenia em idosos, mas é importante ressaltar que uma baixa ingestão como $(0,8 \mathrm{~g} / \mathrm{kg} / \mathrm{dia})$ não vai apresentar grandes resultados. Então, se faz necessário uma ingestão de $(1,2$ e 1,5g/kg/dia) para que a suplementação apresente resultados importantes no combate a sarcopenia. Além disso, a gestão de proteína via oral apresentou grandes resultados no tratamento.

Ao comparar a eficácia da proteína do soro do leite, aminoácidos essenciais, suplementação de vitamina $D$ e placebo na melhoria da forca muscular em idosos sarcopêrnicos em reabilitação dos sexos masculino e feminino com idade acima de 65 anos, os participantes do grupo tratamento apresentaram um aumento significativamente da massa livre de gordura (FFM), melhor atividade de vida diária (ADL), componentes físicos (PCS) e melhor resultado na mini avaliação nutricional (MNA) o grupo placebo não apresentou resultados significativos. ${ }^{12} \mathrm{O}$ grupo tratamento apresentou uma redução no percentual (\%) da gordura android, mão não no grupo placebo. Quanto à proteína C-reativa (CRP) o grupo tratamento apresentou redução da (CRP) e o placebo um significativo aumento. E cerca de
$68 \%$ dos idosos sarcopênicos apresentaram melhora na força muscular.

Afim de uma maior compreensão sobre a relação dos complementos alimentares no manejo nutricional da sarcopenia nos idosos, houve a correlação dos suplementos alimentares Whey Protein, Creatina e Leucina no aumento da musculatura em idosos sarcopênicos respectivamente. A suplementação nutricional com whey protein melhora o índice de massa esquelética relativa, força de preensão manual e melhora a composição física em idosos com sarcopenia ${ }^{13}$, a creatina parece aumentar a resposta adaptativa do músculo ao estímulo do treinamento, por meio do aumento da capacidade para exercícios de maior intensidade e/ou pelo aumento da recuperação e adaptação pós-exercício ${ }^{14}$ e a suplementação com leucina melhora o desempenho funcional medido pelo tempo de caminhada e melhora o índice de massa magra entendendo que, os suplementos citados aumentaram positivamente a saúde, a funcionalidade e a qualidade de vida de inúmeros idosos com sarcopenia, dessa forma, as intervenções nutricionais apresentaram abordagens promissoras nesses indivíduos.

\section{Considerações finais}

Percebe-se que a suplementação de proteína combinado com o exercício físico em idosos com sarcopenia, melhora a força e espessura muscular, além disso, o consumo de whey protein, creatina e leucina trazem benefícios para a saúde, melhorando desempenho físico e funcional dos idosos. Ademais, a atividade física também se mostra eficaz na prevenção e restauração de perda muscular. Parte dos estudos apresentou grandes intervenções nutricionais através dos suplementos alimentares, onde o risco e desenvolvimento da diminuição de massa muscular reduziu de maneira considerável, demonstrando importantes intervenções na prevenção e tratamento da sarcopenia.

O presente estudo possui pequenas limitações, foram observadas algumas variações em relação aos protocolos de suplementação alimentar adotado em cada estudo e isso se dá ao nível de atividade física e a classificação nutricional do idoso.

Espera-se que o presente estudo sirva como espelho para aumentar a busca por mais pesquisas envolvendo a suplementação 
nutricional em idosos. Nos últimos anos os estudos demonstraram importantes intervenções no tratamento da sarcopenia e isso agregará benefício no desenvolvimento da promoção a saúde.

\section{Referências}

1. Candow DG, Forbes SC, Chilibeck PD, Cornish SM, Antonio J, Kreider RB. Variables Influencing the Effectiveness of Creatine Supplementation as a Therapeutic Intervention for Sarcopenia. Front Nutr. 2019 Aug 9;6:124. doi: 10.3389/fnut.2019.00124.

2. Cruz-Jentoft AJ, Baeyens JP, Bauer JM, Boirie $Y$, Cederholm T, Landi F, Martin FC, Michel $J P$, Rolland $Y$, Schneider SM, Topinková $E$, Vandewoude M, Zamboni M; European Working Group on Sarcopenia in Older People. Sarcopenia: European consensus on definition and diagnosis: Report of the European Working Group on Sarcopenia in Older People. Age Ageing. 2010 Jul;39(4):412-23. doi: 10.1093/ageing/afq034.

3. Becky D, LD e Mary Ellen Posthauer. O Papel da Nutrição na Prevenção da Sarcopenia. Today's Dietitian. Vol. 14 N. 9 P. 62, 2012.

4. Robinson, SM, Reginster, JY, Rizzoli, R., Shaw, SC, Kanis, JA, Bautmans, Rueda, R. A nutrição desempenha um papel na prevenção e tratamento da sarcopenia? Nutrição Clínica, 37 (4), 1121-1132. doi: 10.1016 / j.clnu.2017.08.016 5. Genaro Pde $S$, Pinheiro Mde $M$, Szejnfeld VL, Martini LA. Dietary protein intake in elderly women: association with muscle and bone mass. Nutr Clin Pract. 2015 Apr;30(2):2839. doi: 10.1177/0884533614545404.

6. Liguori I, Russo G, Aran L, Bulli G, Curcio F, Della-Morte D, Gargiulo G, Testa G, Cacciatore $F$, Bonaduce D, Abete P. Sarcopenia: assessment of disease burden and strategies to improve outcomes. Clin Interv Aging. 14;13:913-927, maio,2018. doi: 10.2147/CIA.S149232

7. Lanhers C, Pereira B, Naughton G, Trousselard M, Lesage FX, Dutheil F. Creatine Supplementation and Upper Limb Strength Performance: A Systematic Review and MetaAnalysis. Sports Med. 2017 Jan;47(1):163-173. doi: 10.1007/s40279-016-0571-4.

8. Björkman MP, Suominen $\mathrm{MH}$, Kautiainen H, Jyväkorpi SK, Finne-Soveri HU, Strandberg TE, Pitkälä KH, Tilvis RS. Effect of Protein Supplementation on Physical Performance in Older People With Sarcopenia-A Randomized Controlled Trial. J Am Med Dir Assoc. 2020 Feb;21(2):226-232.e1. doi:
9. Aas SN, Seynnes O, Benestad HB, Raastad $T$. Strength training and protein supplementation improve muscle mass, strength, and function in mobility-limited older adults: a randomized controlled trial. Aging Clin Exp Res. 2020 Abril;32(4):605-616. doi: 10.1007/s40520019-01234-2.

10. Park Y, Choi JE, Hwang HS. Protein supplementation improves muscle mass and physical performance in undernourished prefrail and frail elderly subjects: a randomized, doubleblind, placebo-controlled trial. Am J Clin Nutr. 2018 Novembro 1;108(5):1026-1033. doi: 10.1093/ajcn/nqy214.

11. Malafarina $\mathrm{V}$, Uriz-Otano $\mathrm{F}$, Malafarina C, Martinez JA, Zulet MA. Effectiveness of nutritional supplementation on sarcopenia and recovery in hip fracture patients. A multi-centre randomized trial. Maturitas. 2017 Jul;101:42-50. doi: 10.1016/j.maturitas.2017.04.010.

12. Rondanelli M, Klersy C, Terracol G, Talluri J, Maugeri R, Guido D, Faliva MA, Solerte $B S$, Fioravanti $M$, Lukaski $H$, Perna S. Whey protein, amino acids, and vitamin D supplementation with physical activity increases fat-free mass and strength, functionality, and quality of life and decreases inflammation in sarcopenic elderly. Am J Clin Nutr. 2016 Mar;103(3):830-40. doi: 10.3945/ajen.115.113357.

13. Bo $Y$, Liu $C$, Ji Z, Yang $R, A n Q$, Zhang $X$, You J, Duan D, Sun Y, Zhu Y, Cui H, Lu Q. A high whey protein, vitamin $D$ and $E$ supplement preserves muscle mass, strength, and quality of life in sarcopenic older adults: A double-blind randomized controlled trial. Clin Nutr. 2019 Feb;38(1):159-164.

doi: 10.1016/j.clnu.2017.12.020

14. Dolan E, Artioli GG, Pereira RMR, Gualano B. Muscular Atrophy and Sarcopenia in the Elderly: Is There a Role for Creatine Supplementation? Biomolecules. 2019 outubro 23;9(11):642. doi: 10.3390/biom9110642.

15. Martínez-Arnau FM, Fonfría-Vivas $R$, Buigues C, Castillo Y, Molina P, Hoogland AJ, van Doesburg F, Pruimboom L, Fernández-Garrido J, Cauli O. Effects of Leucine Administration in Sarcopenia: A Randomized and Placebocontrolled Clinical Trial. Nutrients. 2020 março 27;12(4):932. doi: 10.3390/nu12040932. 10.1016/j.jamda.2019.09.006. 


\section{Endereço para Correspondência}

Gisele Viana de Moura

Conj. Dirceu Arcoverde II, quadra 162,

apartamento 302- Itararé, Teresina-PI, Brasil

CEP: 64078-034.

E-mail: giseleviana07@gmail.com

Recebido em 03/03/2021

Aprovado em 11/05/2021

Publicado em 27/09/2021 